\title{
Prestige de la hache et haches de prestige
}

Production et usage des tabarzins en Iran et en Inde au xvIII ${ }^{\mathrm{e}}$ siècle

Prestige of the ax and prestige axes

Production and use of tabarzins in Iran and India in the 18th century

\section{Mélisande Bizoirre}

\section{(2) OpenEdition}

\section{Journals}

Édition électronique

URL : http://journals.openedition.org/cel/2077

DOI : $10.4000 /$ cel. 2077

ISSN : 2262-208X

\section{Éditeur}

École du Louvre

Référence électronique

Mélisande Bizoirre, "Prestige de la hache et haches de prestige », Les Cahiers de l'École du Louvre [En ligne], 13 | 2019, mis en ligne le 17 juin 2019, consulté le 17 septembre 2019. URL : http:// journals.openedition.org/cel/2077; DOI : 10.4000/cel.2077

Ce document a été généré automatiquement le 17 septembre 2019.

\section{c) $($ ) $\odot$}

Les Cahiers de l'École du Louvre sont mis à disposition selon les termes de la licence Creative Commons Attribution - Pas d'Utilisation Commerciale - Pas de Modification 4.0 International. 


\section{Prestige de la hache et haches de prestige}

Production et usage des tabarzins en Iran et en Inde au XVIII ${ }^{\mathrm{e}}$ siècle

Prestige of the ax and prestige axes

Production and use of tabarzins in Iran and India in the 18th century

Mélisande Bizoirre

\section{NOTE DE L'AUTEUR}

Nous remercions vivement Mounia Chekhab-Abudaya pour nous avoir permis d'accéder aux objets du museum of Islamic art de Doha et pour son soutien précieux, Mastaneh Tajbakhsh, Azadeh Samii et Bassam Dayyoub pour leur aide dans la lecture des inscriptions, et Vanessa Rose pour sa relecture minutieuse de cet article et son indéfectible amitié.

Laboratoire : Aix Marseille Univ, CNRS, LA3M, Aix-en-Provence, France.

1 Dans les études islamiques comme dans l'histoire de l'art occidentale, la question des armes ornées est souvent considérée comme périphérique et laissée à des spécialistes de l'armement. L'Iran moderne ne fait pas exception, la mystique ou une histoire prestigieuse étant souvent le seul moyen pour ces objets de trouver leur place dans les expositions ou les monographies. C'est pourtant se priver là d'un support riche et pertinent, qui répond à des besoins d'apparat et participe de rituels sociaux et curiaux. 
Fig. 1

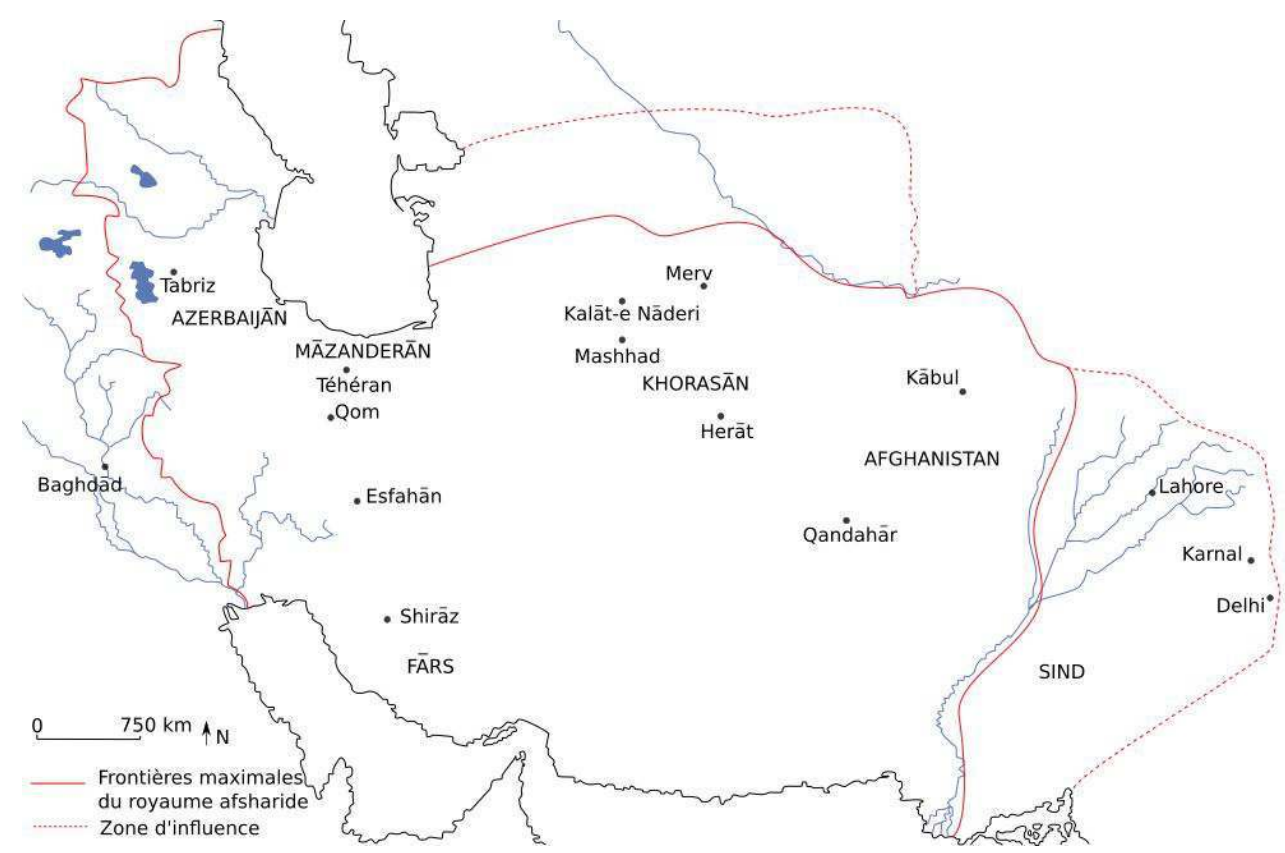

Empire de Nāder Shāh Afshār (1736-47) avec ses principaux centres urbains.

(C) Mélisande Bizoirre

2 L'importance des armes est d'autant plus marquée au XVIII ${ }^{\mathrm{e}}$ siècle que l'Iran connaît le chaos. Tombée en 1135/172 ${ }^{1}$ aux mains d'envahisseurs afghans, la brillante capitale de la dynastie safavide (907/1501-1148/1736), Esfahān, est reprise en 1142/1729. Mais Tahmāsp II qui monte alors sur le trône, n'est guère plus qu'une marionnette aux mains d'un général d'origine obscure, Nāder Qoli Afshār, qui finit par prendre le pouvoir personnellement en 1148/1736 sous le nom de Nāder Shāh. Grand conquérant, il met Delhi à sac en 1152/1739, avant de se tourner vers l'ouest, avec des succès moins marqués.

Originaire du Khorāsān, zone nord-est du monde iranien (fig. 1), Nāder met en valeur sa région natale et sa nouvelle capitale, Mashhad. Son assassinat en 1160/1747 marque l'ouverture de plusieurs années de guerre civile, avant qu'un nouveau souverain, lui aussi d'origine obscure, Karim Khān Zand (r. 1163/1750-1193/1779), ne parvienne à rétablir l'ordre. Il s'installe à Shirāz, dans le sud de l'Iran, mais l'ancien territoire de Nāder reste divisé entre plusieurs potentats : la dynastie Zand (1163/1750-1209/1794) au centre; les Afshārides, successeurs de Nāder, dans le Khorāsān; le tout nouveau royaume d'Afghanistan, créé en 1160/1747 ; des rois indépendants sous influence russe et ottomane en Géorgie et en Arménie. À la fin du XVIII ${ }^{\mathrm{e}}$ siècle, dans de violentes guerres intestines, les shāhs (rois) qājārs Āqā Mohammad Shāh (r. 1193/1779-1212/1797) et Fath 'Ali Shāh (r.1212/1797-1250/1834), réunifient le royaume, au prix de pertes territoriales et du maintien d'un Afghanistan autonome. Leur dynastie conserve le pouvoir en Iran jusqu'en 1344/1925.

Dans une période aussi conflictuelle, dominée non plus par des souverains de palais, éduqués aux arts et à la calligraphie, mais par des militaires ayant conquis leur trône par le sabre, les armes ne pouvaient qu'être le lieu privilégié de l'expression du pouvoir. C'est le cas des tabarzinsou haches d'arçon, connues par la littérature, (تبرزين) au moins depuis le Shāh Nāmeh (v. 1000), mais remises à l'honneur par Nāder Shāh, 
comme le prouvent à la fois les historiographies persanes ${ }^{2}$, leurs illustrations ${ }^{3}$, et les récits de voyageurs :

"The battle-ax is one of the most antient weapons of the Orientals, but it had been for some years much neglected in Persia ; Nader restored the use of it in a more particular manner: it was his favorite weapon, insomuch that before he assumed the diadem, he was generally stiles the Ax-Khan. Afterwards he was often seen with a battle-ax in his hand, playing with it in his tent of audience. His blows with this weapon carried with them inevitable death, of which he gave many proofs." 4

L'identification des tabarzins a déjà été mise en valeur dans un article d'A. S. MelikianChirvani en $1979^{5}$. Celui-ci publiait notamment un groupe de quatre haches signées Lotf 'Ali, datées entre 1148/1735-36 et 1153/1740-41 et présentant des caractéristiques communes. Concernant leur forme (fig. 2), les fers, de 13 à $15 \mathrm{~cm}$ de long, relativement étroits, ont une tranche inférieure qui s'évase légèrement, et leur douille est nettement séparée du tranchant par une moulure. Deux techniques de décor sont principalement utilisées: le champlevé ${ }^{6}$ et les incrustations d'or en inlay ${ }^{7}$. L'iconographie utilise le répertoire de la chasse, avec quelques éléments végétaux récurrents : la vigne habitée d'oiseaux, parfois crachée par un dauphin européanisant; les rinceaux à palmettes bifides entourés de points; des paysages soutenus par une rangée de monticules, où prennent place des arbres à touffes circulaires. Dans son article, A. S. Melikian-Chirvani liait le mélange d'une iconographie iranienne et des rinceaux de vigne d'inspiration européenne à la campagne indienne de Nāder Shāh, sans prendre en compte que ces armes pouvaient porter une datation antérieure à l'événement.

Fig. 2

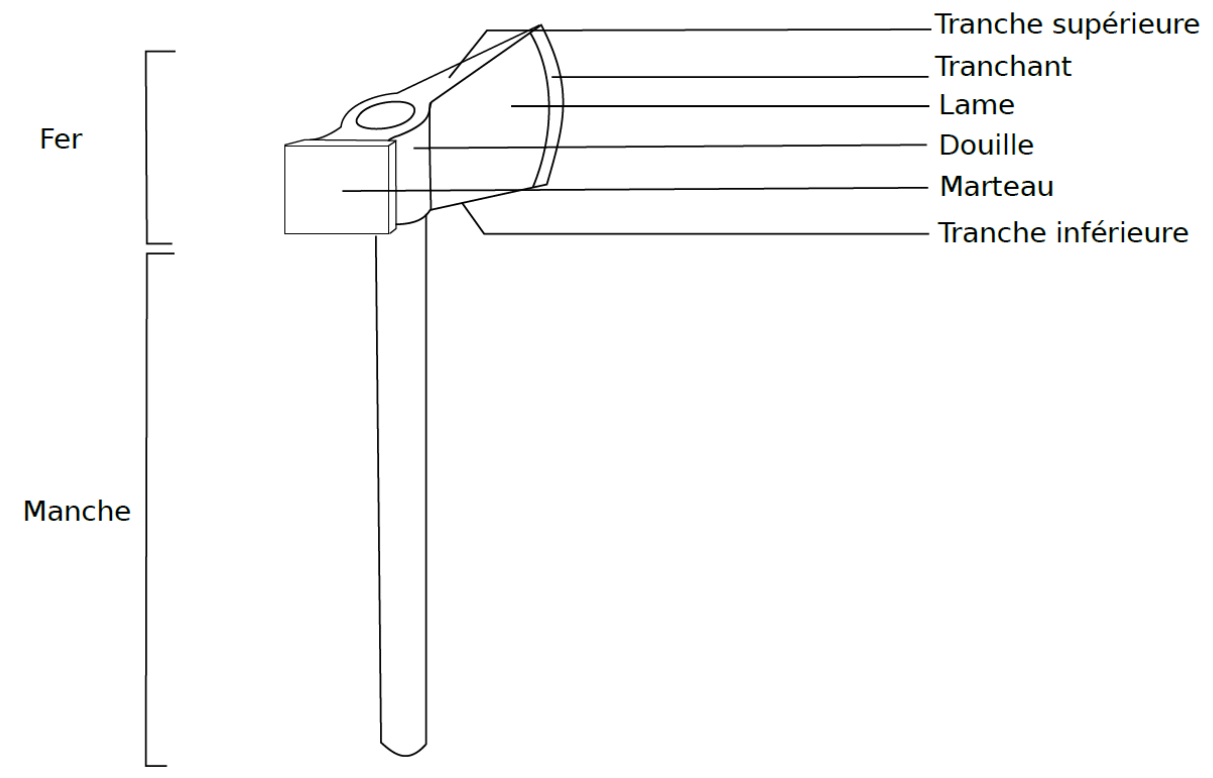

Éléments constitutifs d'une tabarzin (hache d'arçon).

(C) Mélisande Bizoirre

Depuis cette étude de 1979, aucun travail n'a pris la peine de considérer ces tabarzins dans leur ensemble, malgré l'apparition d'un grand nombre de nouveaux exemplaires dans des ventes publiques ou dans des collections russes ou orientales. Les notices rappellent systématiquement la production de Lotf 'Ali, pour lui rattacher l'exemplaire 
considéré, ou pour le dater plus tardivement si la qualité semble inférieure; elles leur attribuent aussi souvent des connotations mystiques ${ }^{8}$.

En nous appuyant sur quelques exemples datés ou facilement datables, qui ne représentent qu'une partie de cette production, nous tenterons au contraire de mettre en valeur l'existence, au xvIII ${ }^{e}$ siècle, d'une pluralité de centres de productions et de plusieurs artistes majeurs, constituant parfois des ateliers familiaux, et de préciser la fonction de ces objets.

\section{La production de Mohammad Taqi à Lahore}

7 Les deux tabarzins datées les plus anciennes que nous connaissions appartiennent à une collection privée danoise ${ }^{9}$ et sont signées d'un certain Mohammad Taqi. Bien que l'une des deux ait un tranchant plus large que les haches d'arçon ordinaires, leur identification est indiscutable car chacune porte inscrit un distique du poète Hatefi (m. 927/1521) en l'honneur de la tabarzin. L'une est datée 1138/1725-26 et toutes deux portent une dédicace au (Sarkar) Khodāyār Khān 'Abbāsi. Ce titre peut s'appliquer à plusieurs hauts personnages du Sind, province du nord-est de l'empire moghol, mais la datation permet d'identifier le dédicataire à Nur Mohammad Kalhora, gouverneur de Lahore de 1719 à $1755^{10}$. L'existence d'un atelier de production dans cette ville est par ailleurs attestée par une tabarzin différente et non-datée, qui porte la mention «fait à Lahore $»^{11}$.

Sous plusieurs aspects, les deux haches de Mohammad Taqi se distinguent des exemples fabriqués en Iran, en particulier par leur manche en métal, incrusté d'un fil d'argent entouré en spirale ${ }^{12}$ et par leur douille angulaire et non circulaire ${ }^{13}$. Néanmoins, les corrélations avec les productions iraniennes restent fortes. La forme de la hache la plus petite est sensiblement identique à celles de Lotf 'Ali, qui utilise par ailleurs le même distique de Hatefi sur l'une de ses œuvres, avec une variation ${ }^{14}$. Un autre élément commun entre les deux artistes est l'usage des rinceaux de vigne habités d'oiseaux, d'origine probablement occidentale.

\section{Lotf 'Ali et ses suiveurs : un atelier familial à Esfahān ?}

\section{Sous Nāder : Lotf 'Ali gholām}

Cette communauté de formes et de motifs est intrigante, d'autant que plusieurs indices laissent à penser que Lotf 'Ali, bien qu'il ne localise jamais ses productions, travaillait à $2000 \mathrm{~km}$ de là, dans la ville d'Esfahān.

Depuis 1979, son corpus s'est considérablement élargi (fig. 3). On compte désormais neuf tabarzins et deux armures signées, datées entre 1142/1729-30 et 1153/1740-41, soit une période qui commence à la reprise d'Esfahān par les Safavides et s'achève avec le retour de Nāder Shāh de sa campagne en Inde et en Asie centrale. Tous ces objets partagent d'évidentes caractéristiques techniques, formelles et ornementales. Certains d'entre eux présentent des décors mettant en scène personnages et animaux dans des paysages (fig. 4), plus complexes que les décors du premier corpus rassemblé par A. S. Melikian-Chirvani. 
Fig. 3

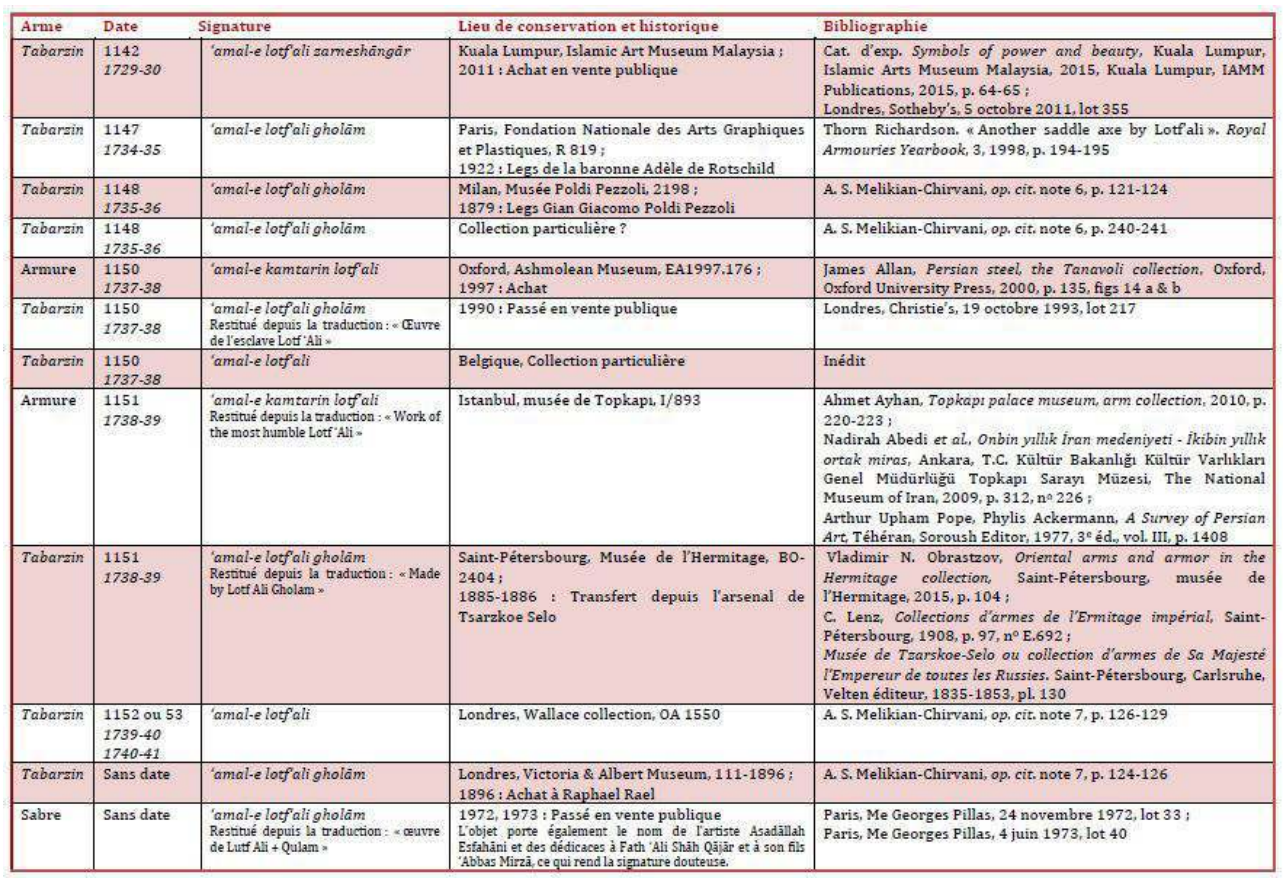

Corpus de Lotf 'Ali gholām (1142/1729-30-1153/1740-41).

(c) Mélisande Bizoirre

9 Ces représentations empruntent de toute évidence à l'art du livre. Elles constituent un premier indice de localisation de l'activité de Lotf 'Ali par leur proximité avec « l'école d'Esfahān ", développée dans un contexte de cour à la fin du XVII ${ }^{\mathrm{e}}$ siècle par des artistes comme Mohammad Zamān, 'Ali Qoli Jebbādār et leurs suiveurs ${ }^{15}$. Les vêtements des cavaliers chasseurs de la hache de la Fondation Nationale des Arts Graphiques et Plastiques (FNAGP) ou de celle de l'Hermitage, une sorte de veste à brandebourgs, un large turban, ou encore un bonnet de fourrure géorgien, rappellent ainsi les costumes des dignitaires de la cour de Shāh Soleimān (r. 1077/1666-1105/1684) représentés par 'Ali Qoli Jebbādar'16. Le chapeau européen (kolāh-e farangi) visible sur les bustes de la tabarzin du Victoria \& Albert Museum ${ }^{17}$ est également fréquent dans ces peintures oocidentalistes, et les touffes circulaires des arbres de Lotf 'Ali trouvent aussi des parallèles chez Mohammad Zamān.

10 L'iconographie de la chasse reste à la mode à la fin du XVII siècle et au début du XVIII ${ }^{\mathrm{e}}$ siècle dans de nombreux supports luxueux : livres, textiles, céramiques, etc. On note que certains des chevaux de Lotf 'Ali manquent d'une pièce de harnachement passant sur le poitrail (martingale), malgré un sens du détail affirmé. Ce trait, qui - à notre connaissance - ne trouve pas d'équivalent dans l'art du livre iranien ni indien, est aussi présent sur un groupe de carreaux à décor de ligne noire à caractère safavide, mais dont l'originalité n'est pas certaine ${ }^{18}$. 
Fig. 4

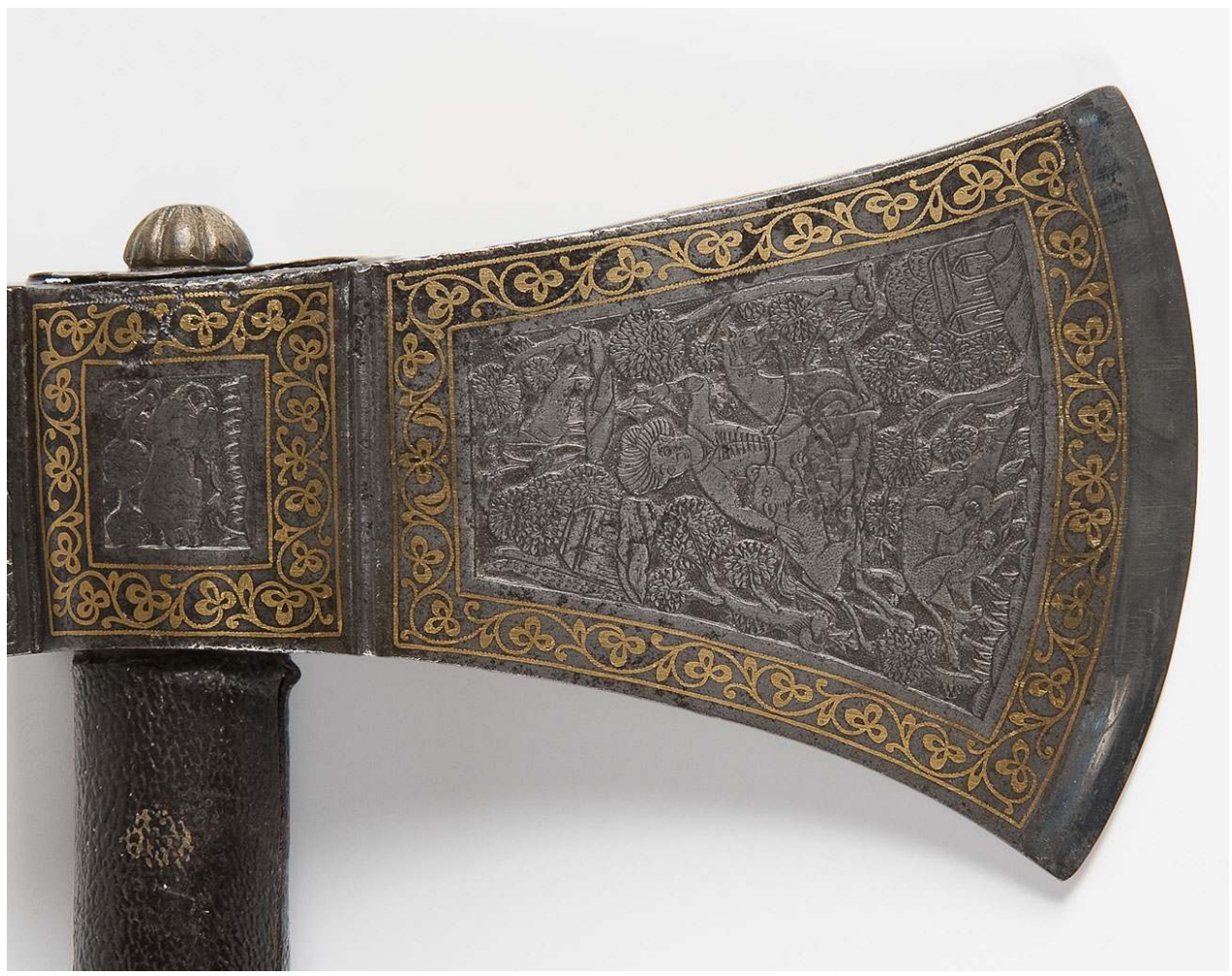

Lotf 'Ali, Tabarzin, acier et or, L. (manche) : $55 \mathrm{~cm}$, L. (fer) : 14,2 cm, Paris, Fondation Nationale des Arts Graphiques et Plastiques, R 819.

(c) Musée du Louvre, Dist. RMN-Grand Palais/Claire Tabbagh/Collections Numériques

11 L'usage du mot "gholāmdans la signature de Lotf 'Ali est un second argument (غلام) " pour une localisation à Esfahān: le terme, signifiant littéralement « esclave », désigne sous les Safavides une caste servile, provenant en grande partie de Géorgie, et constituant une élite militaire ${ }^{19}$. Mis en avant par Shāh 'Abbās (r. 996/1588-1038/1629), le rénovateur de l'État safavide, les gholāms pouvaient aussi servir dans les ateliers royaux, situés essentiellement dans la capitale ${ }^{20}$. Certains fabricants d'armes safavides utilisent d'ailleurs cette mention dans leur nom ${ }^{21}$. Il n'est peut-être pas anodin que l'activité de Lotf 'Ali semble commencer au moment où Esfahān redevient le siège de la cour safavide, et s'interrompe à l'heure où Mashhad tient un rôle prépondérant ${ }^{22}$.

\section{Sous les Zand : Mohammad Rezā ibn Lotf 'Ali}


Fig. 5

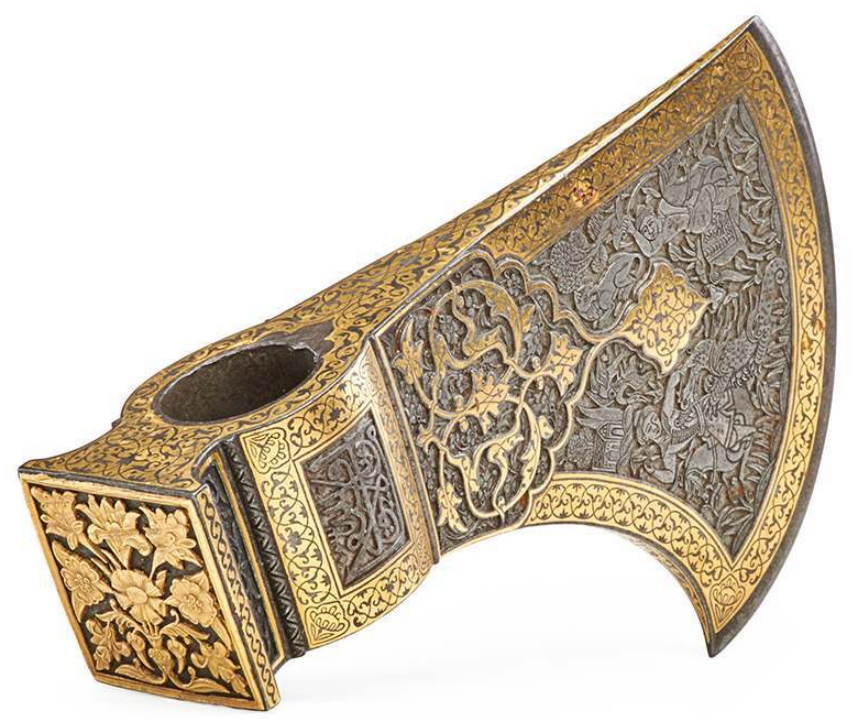

Mohammad Rezā ibn Lotf 'Ali, Fer de tabarzin, 1177/1763-64, acier et or, 12,7 × 7,6 cm, Lambertsville, RAGO, 5 décembre 2015, lot 603

(C) Rago Arts and Auction Center

Un troisième indice de cette localisation de l'atelier de Lotf 'Ali à Esfahān est la poursuite de la fabrication d'armes dans cette ville sur le long terme, attestée par les sources jusqu'à la fin de la période qājāre ${ }^{23}$. Dans la seconde moitié du XvIII ${ }^{\mathrm{e}}$ siècle, deux tabarzins signées d'un certain Mohammad Rezā pourraient aussi témoigner qu'Esfahān reste un centre de production. L'une est passée en vente aux États-Unis chez RAGO en 2015 (fig 5) ${ }^{24}$. Portant un décor narratif nettement inspiré de modèles de Lotf 'Ali jusqu'à reproduire l'absence de martingale de la hache de la FNAGP - elle présente néanmoins quelques différences marquantes: des incrustations d'or plus importantes, en réserve; des scènes narratives plus chargées, moins délicatement regravées; l'apparition de nouveaux motifs floraux, dont un vase jaillissant sur le marteau, qui fait appel à un répertoire connu dans la peinture et sur les laques safavides et postsafavides, en particulier la centaurée centrale ${ }^{25}$.

Cette hache est datée 1177/1763-64 et porte une signature à la lecture difficile. On y déchiffre sans problème le terme 'amalœuvre de ", ainsi que les noms de ", (عمل) Mohammad Rezā et Lotf 'Ali, mais l'ordre et le rapport entre ces mots posent question. Une lecture possible serait 'amal-e Lotf 'Ali ibn Mohammad Rezā shod 1177) عمل لطفعلى (1IVF ) ابن محمد رضا شد (IIVV), "ceci est l'œuvre de Lotf “Ali, fils de Mohammad Rezā") Toutefois, la date tardive, plus de vingt ans après la dernière œuvre signée de Lotf 'Ali, laisse envisager une lecture inverse : Mohammad Rezā ibn Lotf 'Ali (« Mohammad Rezā fils de Lotf 'Ali »).

Cette hypothèse est renforcée par un autre fer conservé au musée d'art islamique de Doha, qui est signé 'amal-e kamtarin / Mohammad Rezā,(عمل كمترين / محمد رضا) 
« œuvre du plus humble / Mohammad Rezā » (fig. 6) ${ }^{26}$. Lui aussi semble dater de la seconde moitié du XVIII ${ }^{e}$ siècle, étant donnée sa dédicace au sāheb ("propriétaire ») Mohammad 'Ali Khān Qājār Dāvānlu ou Qāvānlu/(صاحب محمد على خان قاجار داوانلو (؟) (?) Le nom Mohammad 'Ali Khān n'est pas rare en Iran, et la lecture du nom du clan est difficile, d'autant que les orthographes ne sont pas entièrement fixées à cette époque; il s'agit probablement des Qovānlu ou des Dāvālu, les deux principales branches de la tribu turkmène des Qājārs. Le seul Mohammad 'Ali Khān Qājār qui semble avoir eu des fonctions importantes au XVIII siècle est un Davālu, qui prend part aux luttes entre Qājārs et Zand, puis internes aux Qājārs, dans les vingt dernières années $\mathrm{du} \mathrm{xvIII}^{\mathrm{e}}$ siècle. Il possède justement la charge de gouverneur militaire d'Esfahān ${ }^{27}$.

Fig. 6

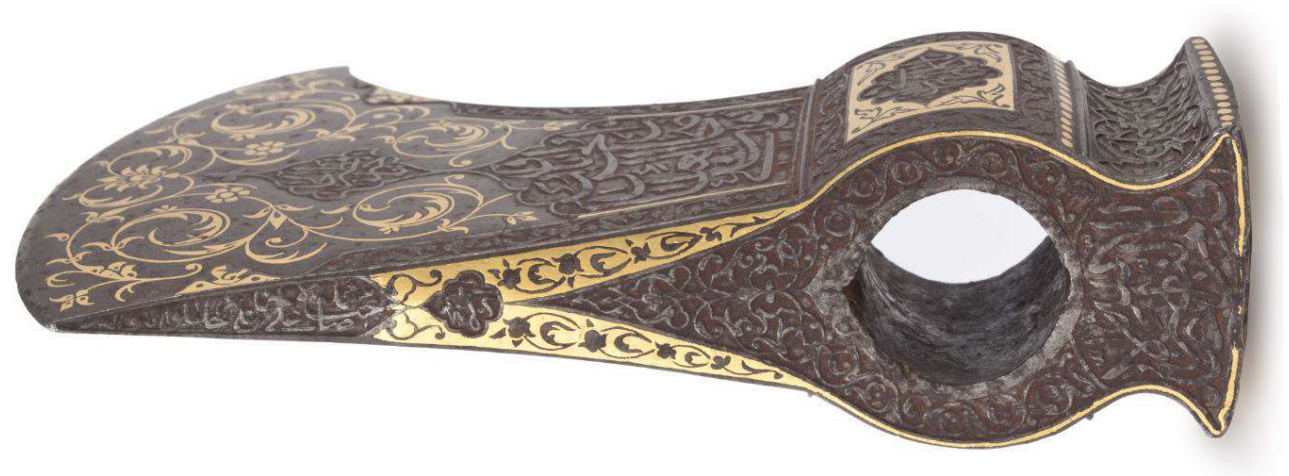

Mohammad Rezā, Fer de tabarzin dédié à Mohammad 'Ali Khān Qājār, non-daté, acier et or, 13,5 × $8 \mathrm{~cm}$, ép. 3,8 cm, Doha, Museum of Islamic art, MIA MW.2014.103.

(c) The Museum of Islamic Art, Doha/Samar Kassab

Cette hache, qui détient un semblable dans la collection Khalili ${ }^{28}$, se distingue par l'emploi, sur son tranchant, d'amples arabesques à feuilles lancéolées, inconnues sur les œuvres de Lotf 'Ali, et qui ne semblent pas connaître de postérité. Les inscriptions en arabe sont des formules religieuses à connotation shiite ${ }^{29}$; on trouve aussi sur la hache de la collection Khalili, le distique de Hatefi, sans la transformation du terme khun en khud. Le fer du musée de Doha (MIA) présente également d'importantes corrélations avec celui de la vente RAGO, notamment dans son usage plus extensif de l'or, avec des décors en réserve, dont des rinceaux à palmettes bifides ponctués. Il semble donc probable que Mohammad Rezā, fils de Lotf 'Ali, ait maintenu à Esfahān, dans la seconde moitié du XVIII ${ }^{e}$ siècle, son activité de facteur d'armes. Deux objets conservés à Téhéran, sans date ni commanditaires, mais présentant des caractéristiques stylistiques proches de celle de la hache RAGO, pourraient également être de sa main ${ }^{30}$. 


\section{'Ali, un autre élève de Lotf 'Ali ?}

Le manche présente un décor d'émaux vert translucide et opaques ${ }^{33}$, selon une technique déjà ancienne : un fusil au nom de Shāh Tahmāsp II, daté 1143/1730-31 présente un décor de fleur assez semblable sur une bague ${ }^{34}$ et le musée de l'Hermitage conserve un poignard dont le fourreau porte un décor floral émaillé en vert translucide et émaux opaques, avec, en partie supérieure, un couple dont l'homme porte un couvre-chef typique du règne de Nāder Shā $h^{35}$. Quelques autres exemples d'objets émaillés provenant notamment du même don d'Hosein Qoli Khān témoignent d'une poursuite de la pratique au cours du xvIII siècle $^{36}$.

Le décor du fer de la hache de Qom se rapproche beaucoup des productions de Mohammad Rezā. La bordure de la douille est presque exactement la même que celle de l'une des haches du musée militaire de Téhéran, à la différence que dans les coins prennent place de larges feuilles, et non des lis ${ }^{37}$; les rinceaux qui ornent la base de son tranchant rappellent aussi le marteau des deux haches de Téhéran, et plus encore le tranchant de la hache de la vente RAGO. Des parallèles existent également avec la hache de Doha, en particulier une large fleur à sept pétales. L'inscription utilisée, classique, invoque un nom de Dieu; on la trouve sur une hache de Lotf 'Ali ${ }^{38}$. La possibilité d'un produit du même atelier n'est donc pas à exclure; on peut aussi envisager que 'Ali soit l'émailleur, et Mohammad Rezā le forgeron. Seul un accès à l'objet et à sa signature permettrait de confronter ces hypothèses.

Si ces différents éléments semblent corroborer l'hypothèse d'un atelier familial iranien probablement situé à Esfahān, ils n'expliquent pas la communauté de motifs et de formes sensible entre les productions de Lotf 'Ali et celle de Mohammad Taqi, à Lahore. Plusieurs hypothèses sont envisageables, en premier lieu un déplacement d'artiste. Il n'est pas impossible, en particulier, que Mohammad Taqi ait été originaire d'Iran et ait émigré au moment des troubles de 1134-35/1722, puisqu'on connaît un sabre signé d'un nom proche, Mohammad Taqi Sakkak ${ }^{39}$, daté 1106/1694-95 et dédié au souverain safavide Shāh Soltān Hosein ${ }^{40}$. Il est possible également que les deux facteurs aient utilisé des sources d'inspiration communes. Les relations entre Inde et Iran et les transferts de modèles ne commencent pas avec Nāder Shāh : un important commerce relie les deux empires depuis plusieurs siècles, en particulier par la route de Qandahar et Lahore. Le motif de vigne n'a pas attendu la campagne de Nāder pour prendre place dans l'art du livre iranien ${ }^{41}$; on en trouve un exemple stylistiquement très proche des décors de Lotf 'Ali sur un décret de Shāh Tahmāsp II daté de 1143/1730-31'22. Il n'est pas certain d'ailleurs que la vigne fasse partie des éléments européens ayant transité par l'Inde avant d'atteindre l'Iran: elle décore des colonnes torsadées dans de grandes huiles sur toile datables de la fin de la période safavide, qui semblent avoir emprunté aux artistes arméniens et géorgiens présents à Esfahān - si elles ne sont pas directement leur œuvre ${ }^{43}$. 


\section{Production indienne pour Nāder Shāh}

Fig. 7

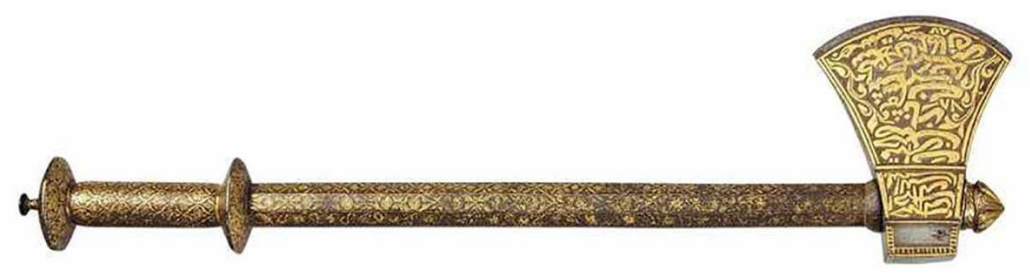

Tabarzin dédiée à Nāder Shāh, v. 1152/1739, acier et or, L. (manche) : 52 cm, Kuala Lumpur, Islamic art museum of Malaysia

(c) Kuala Lumpur, Islamic art museum of Malaysia

Les productions indiennes sont parfois bien plus éloignées des canons esthétiques iraniens, comme en témoigne un troisième ensemble constitué de deux haches indiennes au nom de Nāder Shāh (fig. 7) ${ }^{44}$.

Dotées d'un manche en acier incrusté d'or dont la poignée, encadrée par deux éléments en forme de soucoupe, rappelle les gardes de sabres tulvar, elles possèdent un fer qui s'évase de manière symétrique, divisé en trois parties, avec un marteau plus simple et plus petit que ceux des objets évoqués supra. Toutes deux portent, sur l'une de leur face, la même inscription, dans une calligraphie assez inhabituelle. Sur l'autre face, l'œuvre du musée national de Delhi présente une inscription à caractère religieux, tandis que celle de Malaisie, est couverte de scènes figurées aux protagonistes dorés autour de l'épée de 'Ali, zhu'l faqar. L'iconographie est, sans ambiguïté, indienne : deux tigres, une chasse au tigre et un cornac faisant face à son éléphant.

Sur les deux objets, les inscriptions font clairement référence à Nāder Shāh : be hast

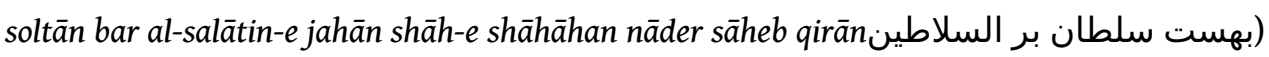
جهان شاه شاهان نادر صاحب قران), "cest le sultan au-dessus des sultans du monde, le roi des rois, Nāder sāheb qirān ». Cette titulature est présente sur un monnayage émis en Iran entre 1152/1739-40 et 1160/1747 (Darband, Esfahān, Mashhad, Shirāz, Tabriz) et dans le Sind (Ahmadābād, Bhakkar, Multān, Peshawār, Sind) en 1152/1739-40, soit au moment de la campagne indienne ${ }^{45}$. L'usage d'un modèle monétaire explique peut-être la calligraphie : on retrouve sur une pièce au nom de Shāh Soleimān la même ligature entre le alef (I) et le he (o) du mot shāh (شاه) d'armes fabriquées dans le Sind ou au nord de l'Inde pour le conquérant. Le centre de Lahore vient évidemment à l'esprit, mais il existe une grande différence, esthétique, formelle et qualitative, avec les exemples faits par Mohammad Taqi pour Khodāyār Khān 'Abbāsi. Il n'est pas impossible qu'il s'agisse d'une production d'un autre centre, non localisé ${ }^{47}$, à moins que celui de Lahore ait été suffisamment important pour produire des objets d'une grande diversité. 
La production indienne du milieu du XVIII ${ }^{e}$ siècle est en effet marquée par une très grande variété des formes et des décors, comme en témoignent d'autres exemples : une tabarzin rapportée par le major-général Robert Clive, en Inde entre 1744 et 1767, au manche en métal tripartite et au fer orné de lotus enfermés dans un treillis, doté d'un étui de cuir ${ }^{48}$; une autre destinée à Safdar Jang Bahadur, établi gouverneur de Lucknow et de la province d'Awadh par l'appui de Nāder Shāh en 1739, et démis en 1753, deux ans avant sa mort ${ }^{49}$; ou encore un modèle à marteau étréci, représenté sur un portrait moghol d'un souverain afghan (probablement Ahmad Shāh Dorrāni ${ }^{50}$ ) et dont on connaît au moins un exemplaire passé en vente ${ }^{51}$. On peut se demander si des armes de type semblable, mais aux formes et décors si différents, témoignent davantage de la vitalité d'un même centre, ou plutôt d'une production géographiquement très répandue.

Ces quelques tabarzins iraniennes et indiennes, choisies pour leur facilité de datation, sont loin de représenter l'ensemble d'une production protéiforme, marquée par les transferts, les copies et les inspirations ${ }^{52}$. Elles montrent néanmoins le maintien d'une tradition vivace de facture d'armes de luxe tout au long $\mathrm{du} \mathrm{xVIII}^{\mathrm{e}}$ siècle, période trop souvent négligée et considérée comme an-artistique. Guerrières et prestigieuses, portant des invocations à la victoire, elles sont alors destinées aux souverains et à une haute aristocratie, comme le prouvent, textes, représentations ${ }^{53}$ et mentions de commanditaires sur les objets eux-mêmes ${ }^{54}$.

$\mathrm{Au}$ cours de la période qājāre, il est probable que, comme l'avance F. Çakir Phillip, les sufis s'en soient emparés et les aient détournées de leurs fonctions originelles pour en faire un symbole de combat spirituel, tandis que l'armée subissait d'importantes modernisations ${ }^{55}$ et qu'une nouvelle esthétique du pouvoir se développait ${ }^{56}$. La hache est en effet depuis bien longtemps l'attribut du mystique et la production semble en effet se poursuivre tout au long du xIX siècle, bien que les tabarzin ne soient alors plus, pour l'essentiel, ni datées, ni attribuées. Une photographie de dervish tenant une de ses armes sur ses genoux, attribuable à la fin du $\mathrm{XIX}^{\mathrm{e}}$ ou au début du $\mathrm{xx}^{\mathrm{e}}$ siècle atteste de ce changement de fonction ${ }^{57}$.

Il serait réducteur, pourtant, de considérer que la tabarzin ne possède, au xix siècle, qu'une symbolique mystique, d'autant que les représentations de dervish, notamment celles de Nur 'Ali Shāh, sont généralement associées à des armes plus longues et au fer plus large. Au contraire, en 1218/1803-04, le peintre Mehr 'Ali en associe une au souverain légendaire Jamshid (fig. 8) ${ }^{58}$ et on en retrouve une autre sur un portrait de premier ministre (sadr-e a zam) qājā̄ ${ }^{59}$. Un cliché datant probablement des années 1880 montre encore un chef de famille trônant fièrement avec sa tabarzin ${ }^{60}$, preuve du maintien du caractère prestigieux de ces armes. 
Fig. 8

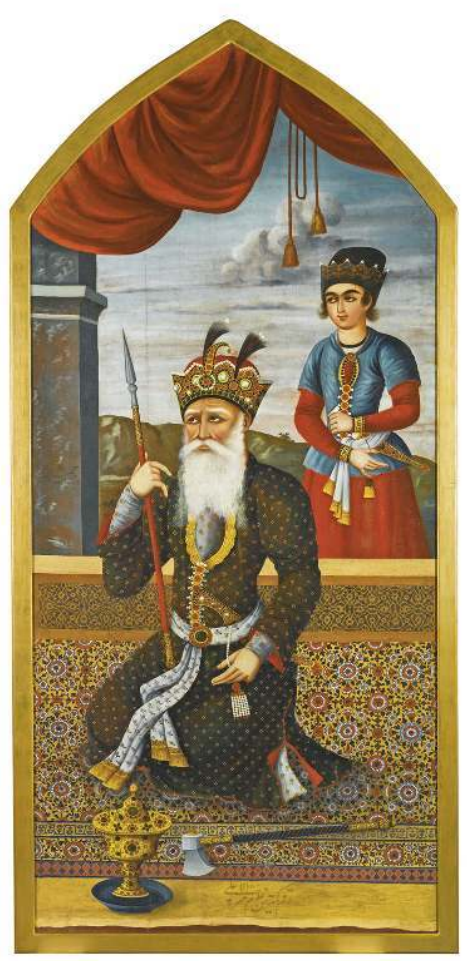

Mehr 'Ali, Le roi Jamshid, 1218/1803-04, huile sur toile, 302 x 145 cm, Londres, Sotheby's, 7 octobre 2015, lot 300

(c) Photo courtesy of Sotheby's, 2019

Quelques jours avant la publication de cet article, un nouveau fer de tabarzin signé « 'amal-e lotf'ali $1150 »$ est passé en vente [Thurcoft (Grande-Bretagne), Paul Beighton Auctioneers, 4 mars 2019, lot 427]. Cohérent iconographiquement et techniquement avec le reste du corpus, il semble cependant devoir être considéré comme une copie étant donné sa qualité de réalisation plutôt moyenne.

\section{NOTES}

1. Nous mentionnons la date hégirienne lunaire (calendrier " arabe ») et la date grégorienne.

2. Sur l'emploi du terme tabarzin dans les récits de la période de Nāder Shāh, voir Manouchehr Khorasani, Lexicon of arms and armor from Iran, a study of symbols and terminology, Tübingen, Legat, 2010.

3. Mirzâ Mohammad Mehdi Esterâbâdi, Tarikh-e Jahângoshây-e Nâderi, illustrated manuscript of 1757, Téhéran, Soroush Press \& Negar Books, 1991, p. 147 ; Mohammad Kāzem, Nāmeh-ye 'ālam ārā-ye nāderi, Moscou, izdatel'stvo vostočnoj literatury, 1960-1965, vol. 1, p. 409.

4. «La hache de guerre est l'une des armes les plus anciennes parmi les orientaux ; mais elle a été fort négligée pendant plusieurs années en Perse; Nader en a rétabli l'usage, d'une manière particulière : c'était son arme favorite, au point qu'avant qu'il ne coiffe le diadème, il était appelé 
"le khan à la hache". Par la suite, il fut souvent vu une hache de guerre à la main, jouant avec dans sa tente d'audience. Un coup de lui avec cette arme amenait une mort certaine, ce dont il donna de nombreuses preuves. » (traduction de l'auteur)

Jonas Hanway, The Revolutions of Persia, containing the reign of Shah Sultan Hussein; the Invasion of the Afghans, and the reigns of Sultan Mir Maghmud and his successor Sultan Ashreff; with the history of the celebrated usurper Nadir Kouli, from his birth in 1687, 'till his death in 1747, and some particulars of the unfortunate reign of his successor Adil Shah, Londres, T. Osborne, D. Brown, etc., 1762, vol. 2, p. 437.

5. Assadullah Souren Melikian-Chirvani, « The tabarzinns of Loțf'alī », dans R. Elgood (éd.), Islamic Arms and Armour, Londres, Scholars Press, 1979, p. 117-135, 240-241.

6. Le fond des décors champlevé est parfois couvert d'un fin piquetage, peut-être préparatoire à une dorure aujourd'hui disparue.

7. L'incrustation en inlay suppose le creusement préalable d'une gouttière permettant d'incruster ensuite le fil ou le bord de la plaque de métal précieux, à l'inverse de l'overlay, qui relève davantage de la dorure. Ces techniques sont décrites par plusieurs sources du XIX ${ }^{\mathrm{e}}$ siècle, dont les deux plus importantes sont Julien de Rochechouart, Souvenirs d'un voyage en Perse, Paris, Challamel aîné, 1867, p. 233-239 et L.-J. Olmer, " Rapport sur une mission scientifique en Perse ", dans Nouvelles archives des missions scientifiques et littéraires, choix de rapports et instructions, Paris, Imprimerie nationale, 1908, t. XVI, fasc. 1, p. 1-110, p. 88. Pour un point sur les sources et les techniques, voir Manouchehr Khorasani, Arms and armour from Iran, the bronze Age to the end of the Qajar period, Tübingen, Legat Verlag GmbH \& Co, 2006, p. 183.

8. Voir par exemple Filiz Çakir Philip, « Hache (tabarzin) », dans cat d'exp. L'Empire des roses. Chefs d'ceuvre de l'art persan du XIX siècle, sous la direction de Gwenaëlle Fellinger, Lens, musée du Louvre-Lens, Gand, éditions Snoeck, 2018, nº 96, p. 108.

9. Cat. d'exp. Islamiske våben $i$ dansk privateje, Copenhague, Davids Samling, Copenhague, Udstilling på Davids Samling, 1982, $\mathrm{n}^{\circ} 80$ p. 122-123 et $\mathrm{n}^{\circ}$ 81, p. 124-125.

10. «Khodāyār Khān » est une titulature employée depuis au moins le XIV siècle, période où un homme portant ce titre compose un traité de fauconnerie. (D. C. Philott (éd.), The Qawānin'sSayyad of Khudā Yār Khān 'Abbāsi, Calcutta, Asiatic Society, 1908). Il est ensuite repris par les Moghols. 'Abbasi est un nom de tribu. Nur Mohammad Kalhora, qui règne sur le Sind entre 1719 et 1755, est mentionné sous le titre de « Khudayar Khan, gouverneur de Pekier [Bhakkar] »dans Jonas Hanway, op. cit. note 4, vol. 2, p. 393 et en tant que «Khudá yár Khán 'Abbási » dans la chronique de An'nand Ra'm Mukhlis (H. M. Elliot, J. Dowson, The History of India as told by its own historians, vol. VIII, Londres, Trübner \& Co, 1877, p. 94-97). L'historien L. Lockhart le considère comme le gouverneur du Sind (Laurence Lockhart, Nadir Shah, Londres, Luzac \& Co., 1938, p. 158). 11. Londres, Victoria and Albert Museum, inv. I.S. 1500 (A. S. Melikian-Chirvani, op. cit. note 5, p. 131-132, pl. 146-147).

12. Les manches iraniens possèdent un manche de bois, entouré d'un fil en spirale sur lequel est moulée une feuille de cuir, et sont parfois dotés d'une feuille de métal à leurs extrémités.

13. Les douilles angulaires sont présentes sur d'autres haches, au tranchant souvent plus large et en général attribuées à l'Inde (Leeds, Royal Armouries, XXVIC.33 et XXVIC.39; Paris, musée du Louvre, R 864 ; Londres, Sotheby's, 18 avril 1984, lot 374 (dédiée à un certain sarkar Munshir Mohammad Khān) ; Londres, Sotheby's, 13 avril 1988, lot 191.). Il existe toutefois un exemple sans doute iranien, signé Shāh Najaf et daté 1201/1796-97. J. Allan, Persian steel, the Tanavoli collection, Oxford, Oxford University Press, 2000, the G.12, p. 316-317.

14. Lotf 'Ali transforme le terme khun (خون), «sang » en khud (خود), « casque ». A. S. MelikianChirvani, op. cit. note 5, p. 240-241.

15. Chahryar Adle, Écriture de l'union: reflets du temps des troubles: cuvre picturale (1083-1124/1673-1712) de Hâji Mohammad, Paris, Nobele, 1980 ; Axel Langer, « European influences on seventeenth-century Persian painting: of handsome Europeans, naked ladies and Parisian timepieces ", dans cat. d'exp. The Fascination of Persia, sous la direction d'Axel Langer, Zürich, 
2013-2014, Zürich, musée Rietberg and Verlag Scheidegger \& Speiss AG, 2013, p. 170-237 ; N. Habibi, Ali Qoli Jebâdâr et l'Occidentalisme safavide, une étude sur les peintures dites farangi sâzi, leurs milieux et commanditaires sous Shâh Soleimân (1666-94), Leyde, Brill, 2017.

16. 'Ali Qoli Jebbādar, "Shāh Soleimān et ses courtisans dans une véranda ", Album de SaintPétersbourg, Iran, probablement Esfahān, v. 1670-1675, peinture: $28,2 \times 42,1 \mathrm{~cm}$, SaintPétersbourg, Institute of Oriental Manuscripts, E-14, fol. 98r.

17. A. S. Melikian-Chirvani identifiait également un turban indien $d u x v I^{e}$ siècle. Celui-ci est connu des peintres de la fin du XVII' siècle : cf. Shāh 'Abbās reçoit un ambassadeur indien, Shaikh 'Abbāsi, 1065/1654-55, Zürich, musée Rietberg, RVA 1039 (Cat. d'exp. The Fascination of Persia, sous la direction d'Axel Langer, Zürich, 2013-14, Zürich : musée Rietberg and Verlag Scheidegger \& Speiss AG, 2013, p. 200, cat.115) ou la fresque sur le même thème au palais Chehel Sotun à Esfahān.

18. Par exemple Los Angeles, LACMA, M 2004.34a-e; Kuala Lumpur, Islamic Art Museum Malaysia, inv. 2007.4.1. Ces carreaux pourraient être soit une œuvre originelle du XVII ${ }^{\mathrm{e}}$ siècle, soit une copie du XIXe siècle d'une œuvre safavide. Nous remercions Lucile Martinet, qui mène un doctorat sur la céramique à décor de ligne noire, pour cette information.

19. Sussan Babaie et al., Slaves of the Shah, new elites of Safavid Iran, Londres, New York, I. B. Tauris, 2004.

20. Mehdi Keyvani, Artisans and guild life in the later Safavid period, contributions to social-economic history of Persia, Berlin, Klaus Schwartz, 1982.

21. C'est par exemple le cas d'un Salmān Gholām sur un sabre dédié à Shāh Sāfi (r. 1038/1629-1052/1642; il peut également s'agir de Shāh Soleimān, celui-ci ayant commencé son règne sous le nom de Safi II). M. Khorasani, op. cit. note 7, p. 168.

22. À partir de 1135/1722 et jusqu'à la fin du XVIII ${ }^{\mathrm{e}}$ siècle, Esfahān connaît une chute de sa population, qui fuit les exactions de gouverneurs trop zélés, les réquisitions permanentes d'équipements militaires et de numéraires, et une situation commerciale en berne. Willem Floor, The rise and fall of Nader Shah, Dutch East India Company reports, Washington, Mage Publishers, p. 87 et $\mathrm{s}$.

23. Par exemple J. de Rochechouart, op. cit. note 7, p. 238 ; L.-J. Olmer, op. cit. note 7, p. 85.

24. Lambertsville, RAGO, 5 décembre 2015, lot 603.

25. Isabelle Imbert, La peinture de fleurs persane et indienne de la période moderne (XVI ${ }^{e}-\mathrm{XVII}{ }^{e}$ siècles), thèse de doctorat sous la direction de J. P. Van Staëvel et E. Brac de la Perière, Université ParisSorbonne, 2015, en particulier vol. 2, n ${ }^{\circ} 16$ p. 39 (fleur supérieure non-identifiée), $n^{\circ} 144$, p. 325 (probable centaurée) et $\mathrm{n}^{\circ} 160$, p. 357 (pichet jaillissant).

26. Doha, musée d'art islamique, 2014.103. Cet objet a été acquis en 2014 auprès d'un collectionneur privé iranien. La signature est divisée en deux moitiés, présentes chacune dans un cartouche sur chaque tranche de l'objet.

27. Hormoz Ebrahimnejad, Pouvoir et succession en Iran, les premiers Qâjârs, 1726-1834, Paris, L'Harmattan, 1999, p. 224, 253-254.

28. Londres collection Khalili, MTW 693 (David Alexander, The arts of war, arms and armour of the $7^{\text {th }}$ to $19^{\text {th }}$ centuries, Londres, Nour Foundation, Azimuth, Oxford, Oxford University Press, 1992, cat. 94, p. 152-153). L'incrustation de cet objet semble de moins bonne qualité que celle de l'exemplaire du MIA. L'objet est passé auparavant dans la collection Hales : Robert Hales, Islamic and Oriental arms and armour, a lifetime passion, Robert Hales, 2013, nos 805 et 807, p. 334.

29. On y trouve notamment la formule على الله فى كل الامور تو كلى و بالخمس اصحاب العبا تو Pour tout, je place mon entière confiance en Dieu et je prie par l'intercession des cinq " تلى كلى amis », qui fait référence aux cinq «personnes du manteau » de la tradition shiite : Mohammad, Fatemeh, Ali, Hasan, Hosein.

30. Téhéran, musée militaire, inv. 36 et sans numéro. M. Khorasani, op. cit. note 7, cat. 354, p. 665 et cat. 355, p. 666. 
31. A.D. Tushingham, «Persian enamels » dans actes du colloque The memorial volume of the Vth international congress of Iranian art and archaeology, Téhéran, Isfahan, Shiraz, 1968, publiés sous la direction de M. Y. Kiani, A. Tajvidi, Téhéran : Ministry of the culture and art, 1972, fig. 5.

32. Il ne peut s'agir du Hosein Qoli Khān qui soutint Karim Khān Zand, puisque celui-ci fut assassiné en 1191/1777. Au moins deux Hosein Qoli Khān se signalent lors des guerres de succession après la mort du Shāh Āqā Mohammad Khān Qājār, de 1212/1797 à 1217/1802 : un Qājār, frère de Fath 'Ali Shāh, et un Afshār. Il est probable qu'il s'agisse du premier, forcé un temps par son frère à trouver refuge dans le sanctuaire de Qom. H. Ebrahimnejad, op. cit. note 27, p. 240-258 ; A. Amanat, "Fath-'alī shah qājār », Encyclopaedia iranica, 2012, en ligne, http:// www.iranicaonline.org/articles/fath-ali-shah-qajar-2 [29/04/2018].

33. Sur l'unique photographie en noir et blanc dont nous disposons, il semble que l'émaillage ne concerne que le manche de l'objet, mais que le fer ne porte que des dorures.

34. Istanbul, Musée du palais de Topkap1, I/1535. (Nadirah Abedi et al., Onbin yıllık İran medeniyeti İkibin yıllık ortak miras, Ankara, T.C. Kültür Bakanlığı Kültür Varlıkları Genel Müdürlüğü Topkapı Sarayı Müzesi, The National Museum of Iran, 2009, $n^{\circ} 235$, p. 232).

35. Saint-Pétersbourg, musée de l'Hermitage, BO-194 (Cat. d'exp. Royal Persian paintings, the Qajar epoch, 1785-1925, sous la direction de Layla S. Diba et Maryam Ekhtiar, New York, 1998, New York, Brooklyn Museum of Art, I. B. Tauris, 1998, cat. 20, p. 141-142, avec une bibliographie complète).

36. A. D. Tushingham, op. cit. note 31.

37. Téhéran, musée militaire, 36. M. Khorasani, op. cit. note 7, cat. 355, p. 666.

38. Yā kāfi al-mahamat (يا كافى المهمات), «Ô Toi qui récompenses les efforts ». A. S. MelikianChirvani, op. cit. note 5, p. 240-241.

39. Le terme sakkak pourrait désigner quelqu'un qui frappe la monnaie.

40. M. Khorasani, op. cit. note 7, p. 168.

41. Marges d'une peinture d"Ali Qoli Jobādār datée rajab 1085/septembre 1674, Londres, collection Hosein Afhsar (A. Langer, op. cit. note 15, cat.102, p. 204-20); Reliure de Coran, 1125/1713-14, Boston, Museum of Fine Arts, 19.793 ; Double page enluminée d'un livre de prières signé Ahmad al-Nairizi, 1132/1719-20, New York, The Metropolitan Museum of Arts, 2003.239 (David Alexander, Islamic arms and armor in the Metropolitan museum of art, New York, The Metropolitan museum of art, 2015, fig. 21, p. 63).

42. Erevan, Matenadaran, Dossier 1g, 331 (Raïssa Amirbekyan, La calligraphie et la miniature de l'Orient, (Collection du Matenadaran), album, Erevan, Naïri, 2012, fig. 131).

43. Doha, musée d'art islamique, PA.606.1998 (A. Langer, op. cit. note 15, cat. 139, p. 242-243) ; Londres, Sotheby's, 13 octobre 1999, lot 13-14 et 25 octobre 2017, lot 115-116.

44. Delhi, National Nuseum of India, 58.47/3 ; Kuala Lumpur, Islamic Arts Museum Malaysia (Cat.d'ex., Symbols of power and beauty, Kuala Lumpur, Islamic Arts Museum of Malaysia, 2015, p. $14 ; 2014$, Londres, Christie's, 11 avril 2014, lot 41).

45. Sephen Album, Sylloge of Islamic coins in the Ashmolean Museum, Oxford, Ashmolean Museum, 2001, vol. 9, p. XXIx.

46. Mahmudi non daté au nom de Soleiman. Rudi Matthee, Willem Floor, Peter Clawson, The monetary history of Iran, Londres, New York, I. B. Tauris, 2013, fig. 23.

47. On pense évidemment à Delhi.

48. Powys (Pays de Galles), Powis Castle, National Trust, 1180572.1 et 2.

49. Londres, Victoria and Albert Museum, inv. 400-1875.

50. Bodleian Library, Ouseley Add. 166, fol. $43 \mathrm{v}$.

51. Christie's, 16 décembre 2002, lot 32 . La hache fait partie d'un ensemble d'armes indiennes et ottomanes offertes au comte Enrico Millo en 1912.

52. A. S. Melikian-Chirvani mettait en valeur l'existence d'un faux signé Lotf 'Ali et daté 1150/1737-38, qu'il attribuait à Lahore (A. S. Melikian-Chrivani, op. cit. note 5,P. 129-130). Deux autres exemples peuvent être donnés : une hache du Victoria and Albert Museum (IM 48-1911) 
qui reprend un exemplaire du Louvre peut-être attribuable à Lotf 'Ali ou à son entourage (OA 7091 - cat. exp. L'Empire des roses, chefs d'œuvre de l'art persan du XIX e siècle, sous la direction de Gwenaëlle Fellinger, op.cit. note 8, cat.96, p. 106); trois œuvres dispersées (New York, Metropolitan Museum of Art, 36.25.1794 - D. Alexander, op. cit. note 28, n 96, p. 234-235 ; Doha, Museum of Islamic Art, 2014.102; Londres, Sotheby's, 13 avril 1988, lot 193) reprenant une tabarzin du musée de Topkapı (I/2378 - Ahmet Ayhan, Topkapı palace museum, arm collection, 2010, p. 151), elle aussi potentiellement sortie de l'atelier de Lotf 'Ali, en regard de son cavalier portant un kolāh-e farangi.

53. Voir par exemple ces trois représentations indiennes du souverain afghan Ahmad Shāh Dorrāni (r. 1160/1747-1187/1773) : Bodleian Library, Ouseley Add. 166, fol. 43r ; Berlin, Museum für Islamische Kunst, I 5063, fol. 20v (identification incertaine) ; British Library, Add. Or. 2685.

54. Ainsi, en 1210/1795-96 ou 1215/1800-01 encore, une tabarzin à décor doré est dédiée à un certain Mohammad Mahdi, non identifié. Sotheby's, 30 mai 2011, lot 138.

55. Sur cette question, voir Jean Calmard. "Les réformes militaires sous les Qâjâr (1794-1925) », dans Yann Richard, Entre l'Orient et l'Occident, Adaptation et assimilation des idées et techniques occidentales en Iran, Paris, Éditions de la Maison des sciences de l'homme, 1989, p. 17-42.

56. F. Çakir Phillip, « Armes et armures. Typologies, usages et symboles », dans G. Fellinger, op. cit. note 52, p.142-147. Sur les objets sufis, voir aussi Akbarnia, Leoni, Light of the sufis et Christiane Gruber, «Matériaux mystiques, l'art religieux et dévotionnel de l'Iran qajar », dans Fellinger, ibid., p. 76-83 et notices liées.

57. New York, Brooklyn Museum, 1997.3.142.

58. Londres, Sotheby's, 7 octobre 2015, lot 300.

59. Christie's, 26 avril 1994, lot 96. Le catalogue précise qu'il s'agit du premier ministre de Fath 'Ali Shāh, mais Mirzā Shafi' Māzandarāni n'est que le premier grand vizir à porter le titre d'origine ottomane «Sadr-e A'zam », qui est repris par ses successeurs. La peinture semble plus tardive, et le haut turban ne correspond pas à l'origine tribale du personnage.

60. Antoin Sevruguin, Famille (?), Washington, Freer Gallery of Art, FS-FSA_A.4_2.12.GN.21.07.

\section{RÉSUMÉS}

$\mathrm{Au}$ XVIII ${ }^{\mathrm{e}}$ siècle, alors que le monde iranien connait une instabilité durable, la hache d'arçon (tabarzin en persan) émerge comme un support artistique à part entière, porteur de décors complexes, inspirés de l'art du livre. Mis en évidence dans une étude d'A. S. Melikian-Chirvani en 1979, ce groupe d'armes ornées peut désormais être enrichi, grâce à l'apparition, sur le marché de l'art ou dans plusieurs musées, de nouveaux spécimens. En s'appuyant sur des exemples portant signatures, dates ou dédicaces, cet article met en évidence la diversité des formes, décors et techniques ainsi que l'existence de lignées d'artistes travaillant dans plusieurs centres de productions, dont Lahore et Esfahān sont les plus évidents. Il revient également sur la fonction de ces armes, parfois associées à des connotations mystiques, mais plus souvent symboles de pouvoir.

In the eighteenth century, when the Iranian world was instable, the battle-axe (tabarzin in Persian) emerged as an artistic support; it bore complex decorations, inspired by that art of the book. First studied as such by A. S. Melikian-Chirvani in 1979, this group of decorate arms could henceforth be enriched, thanks to the appearance on the art market of in several museums of 
new specimens. By basing itself on examples bearing signatures, dates or dedications, this article highlights the diversity of forms, decorations and techniques, as well as the lines of artists working in several centres of production, including Lahore and Esfahan. It also examines the function of the arms, sometimes associated with mystic connotations, but most often symbols of power.

\section{INDEX}

Mots-clés : Tabarzin, haches d'arçons, armes ornées, art iranien, XVIIIe siècle, Afsharides, Zands, Qajars, Nader Shah, Lotf 'Ali

Keywords : Tabarzin, battle-axes, decorated arms, Iranian art, eighteenth century, Afsharids, Zands, Qajars, Nader Shah, Lotf ‘Ali

\section{AUTEUR}

\section{MÉLISANDE BIZOIRRE}

Diplômée de deuxième cycle à l'École du Louvre, Mélisande Bizoirre mène une thèse intitulée « Les productions artistiques dans le monde iranien entre 1135/1722 et 1163/1750 » au sein du Laboratoire d'archéologie médiévale et moderne en Méditerranée de l'université d'Aix-Marseille, sous la direction d'Yves Porter. Elle est également chargée de cours à l'École du Louvre en histoire des arts de l'Islam et a contribué à plusieurs projets de vulgarisation à l'Institut du monde arabe.

***

The holder of a master's degree from the École du Louvre, Mélisande Bizoirre is writing a thesis entitled "Les productions artistiques dans le monde iranien entre 1135/1722 et 1163/1750" at the Laboratoire d'Archéologie Médiévale et Moderne en Méditerranée of the Université d'Aix-

Marseille, under the supervision of Yves Porter. She also lectures at the École du Louvre in the history of the arts of Islam and has contributed several popularisation projects at the Institut du Monde Arabe. 